Bianca Ruschel HILlmann ${ }^{1}$

SérGio Murilo StefFens ${ }^{2}$

Alberto Trapan JUNIOR ${ }^{3}$

\section{Relato de Caso}

Palavras-chave

Trombose venosa

Período pós-parto

Trombofilia

Complicações hematológicas na gravidez

Relatos de casos

Keywords

Venous thrombosis

Postpartum period

Thrombophilia

Pregnancy complications, hematologic

Case reports

\section{Trombose de veia renal \\ no puerpério: relato de caso}

\section{Renal vein thrombosis in the puerperium: case report}

\section{Resumo}

Durante a gravidez e o puerpério, existe um estado de hipercoagulabilidade sanguínea e, portanto, de risco para eventos tromboembólicos. A trombose de veia renal é uma condição grave, pouco frequente e de difícil diagnóstico. Este estudo relatou um caso de trombose de veia renal numa puérpera, descrevendo caso clínico, fatores de risco, métodos diagnósticos e o tratamento instituído.

\section{Abstract}

Pregnancy and puerperium are periods of blood hypercoagulability and, therefore, of risk for thromboembolic events. Renal vein thrombosis is a serious and infrequent condition of difficult diagnosis. This study reported a case of renal vein thrombosis in the puerperium, and described the clinical case, risk factors, diagnostic methods, and treatment instituted.
Correspondência

Bianca Ruschel Hillmann Rua Professora Maria Flora Pausewang, $s / n$

CEP: $88036-800$

Trindade (SC), Brasil

Recebido

$15 / 07 / 2015$

Aceito com modificacocoes

09/09/2015

Hospital Universitário Professor Polidoro Ernani de São Thiago - Florianópolis (SC), Brasil.

'Programa de Residência Médica em Ginecologia e Obstetrícia, Hospital Universitário Professor Polidoro Ernani de São Thiago Florianópolis (SC), Brasil.

2Departamento de Ginecologia e Obstetrícia, Centro de Ciências da Saúde, Universidade Federal de Santa Catarina - UFSC Florianópolis (SC), Brasil.

${ }^{3}$ Divisão de Tocoginecologia, Hospital Universitário Professor Polidoro Ernani de São Thiago - Florianópolis (SC), Brasil. 
Introdução

A doença tromboembólica venosa é uma das causas de morbimortalidade na gravidez e no puerpério ${ }^{1-4}$. A gravidez é considerada um estado trombogênico ${ }^{3,4}$ e existe um aumento de cinco vezes no risco de eventos tromboembólicos venosos durante o ciclo gravídico puerperal ${ }^{1,5}-\mathrm{o}$ risco absoluto de desenvolver a doença é de 7 a $25 \mathrm{em}$ 10.000 gestações $^{6,7}$. Os locais mais comuns de trombose venosa durante a gravidez são as veias iliofemorais ${ }^{8}$.

A trombose de veia renal (TVR) é rara e dificilmente ocorre em pacientes que não tenham condições subjacentes, como síndrome nefrótica ou câncer renal $^{9-11}$. Em dois terços dos casos acomete ambas as veias renais ${ }^{11,12}$. Nos casos de unilateralidade, a veia renal esquerda é mais prejudicada ${ }^{9,11,12}$. Em raros casos, estende-se para veia gonadal $(0,4 \%)^{9}$.

A TVR apresenta quadro clínico insidioso e crônico, sendo diagnosticada apenas quando se associa ao embolismo pulmonar ou insuficiência renal, mas pode ser aguda e com quadro clínico mais grave ${ }^{11-13}$. Os casos de TVR agudos costumam cursar com hematúria macro ou microscópica, dor no flanco e disfunção renal ${ }^{11}$. Menos comumente, cursam com sintomas gerais como náusea, anorexia, febre e dor abdominal intensa, podendo simular abdome agudo ${ }^{11}$. Geralmente, não há proteinúria significativa entre as pacientes que não apresentam síndrome nefrótica ${ }^{14}$.

A TVR aguda não costuma estar relacionada com a síndrome nefrótica, estando geralmente associada a trauma, desidratação (em crianças) ou estados de hipercoagulabilidade $^{15}$. As trombofilias são frequentes nesses pacientes, sendo a deficiência de proteína $S$ a mais relacionada?. $\mathrm{Na}$ gravidez e no parto, a tríade de Virchow está presente: o sistema de coagulação está hiperativado, o fluxo venoso está mais lento e há trauma dos vasos (principalmente pélvicos) durante o parto, trauma este que tende a ser maior na cesariana ${ }^{2,12,16-18}$.

A principal alteração no sistema de coagulação é um estado trombótico, especialmente no final da gravidez e no parto ${ }^{16-19}$. Há um aumento importante dos fatores I, V, VII, VIII, IX, X, XII e de von Willebrand e diminuição da atividade fibrinolítica, os quais voltam ao normal logo após o parto ${ }^{2}$. A velocidade do fluxo sanguíneo reduzido durante a gravidez, o nadir, ocorre entre a $34^{a}$ semana e o termo. O fluxo volta ao normal cerca de seis semanas após o parto ${ }^{2}$. A TVR é considerada trombose venosa grave e a sobrevida das pacientes com esta condição é menor do que aquela observada nas pacientes com história de trombose venosa de membros inferiores?.

O objetivo deste trabalho foi relatar um caso de TVR no puerpério afim de discutir os fatores de risco, a investigação diagnóstica e o tratamento dessa condição rara.
Descrição do caso

Mulher, 21 anos, parda, G4P3A1, previamente hígida. Gestação a termo. Foi internada em trabalho de parto e evoluiu com parto vaginal sem lacerações e sem intercorrências. Após 24 horas do parto, iniciou com dor em flanco esquerdo de forte intensidade, de alívio apenas por meio de analgésicos opioides. A paciente reagia com dor a punho percussão da loja renal esquerda e a apalpação de flanco esquerdo, porém sem defesa abdominal. Durante as crises, apresentava sudorese profusa, vômitos e tontura. Manifestou também nesse período picos hipertensivos. Devido à suspeita de ureterolitíase, foram solicitados exames laboratoriais e radiológicos. O exame simples de urina revelou $1+$ de proteínas, células do epitélio renal e hemoglobina. A creatinina sérica era de 1,9 mg/dL. O exame de ultrassonografia (USG) renal e de vias urinárias foi considerado normal.

Realizou-se, então, uma tomografia computadorizada (TC) de rins e de vias urinárias não contrastada, devido ao aumento da creatinina. A TC sugeriu o diagnóstico de trombose de veia renal e evidenciou veia renal esquerda retro-aórtica (variante anatômica). Foi realizada USG com Doppler dos rins e veias renais, que evidenciou rim esquerdo de dimensões aumentadas $\left(186 \mathrm{~cm}^{3}\right)$, com córtex ecogênico e pirâmides hipoecogênicas. Veias gonadal e renal esquerdas com material hipoecogênico em seu interior. Sem fluxo a dopplervelocimetria até a porção retro-aórtica da veia renal. Foi confirmado, assim, o diagnóstico de trombose das veias renal e gonadal esquerdas (Figuras 1 e 2). A proteinúria de 24 horas teve resultado de $370 \mathrm{mg}$ (não nefrótica) e a investigação de trombofilias foi negativa. Os exames realizados para investigação de trombofilias e doenças autoimunes foram: proteína $C$, proteína $S$, resistência à proteína $C$ ativada, fator VIII, antitransglutaminase, FAN, antitrombina III, fibrinogênio, anticardiolipina $\operatorname{IgG}$ e $\operatorname{IgM}$, anticoagulante lúpico, anticorpo anti-DNA, anti-SM, anti-Ro e anti-La. Todos tiveram resultado normal. Paciente negava história prévia de trombose venosa.

Referiu tabagismo por cinco anos, findo há oito meses. De história familiar referia trombose venosa de membros inferiores em três parentes. Foi optado por anticoagulação, com heparina não fracionada subcutânea 20.000 UI 12/12 horas, e varfarina sódica, $5 \mathrm{mg}$ ministrados por via oral ao dia. Não foi utilizada enoxaparina devido à disfunção renal. Iniciou-se também com benzilato de anlodipino, $5 \mathrm{mg}$ via oral ao dia, para controle pressórico. A paciente teve alta no $13^{\circ}$ dia de puerpério, após a relação normatizada internacional (RNI) do tempo de atividade da protrombina (TAP) atingir valor de 2,0 e a heparina ser suspensa.

Após a alta, foi acompanhada nos ambulatórios de hematologia, nefrologia e obstetrícia. Tem adequada adesão ao tratamento e mantém RNI entre 2,0 e 3,0. A função renal retornou ao normal com valor de creatinina de 1,1 


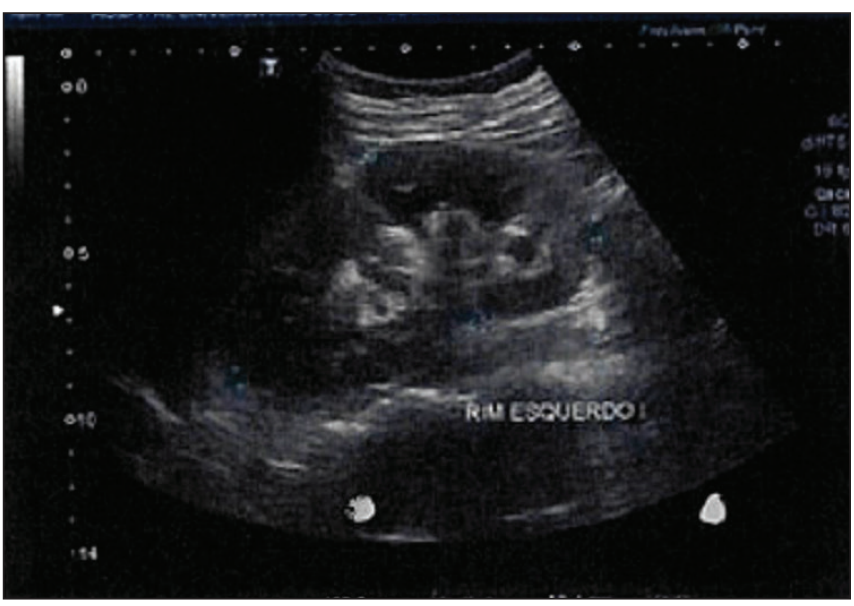

Figura 1. Rim esquerdo apresentando córtex ecogênico e pirâmides hipoecogênicas, sugerindo o diagnóstico de trombose de veia renal

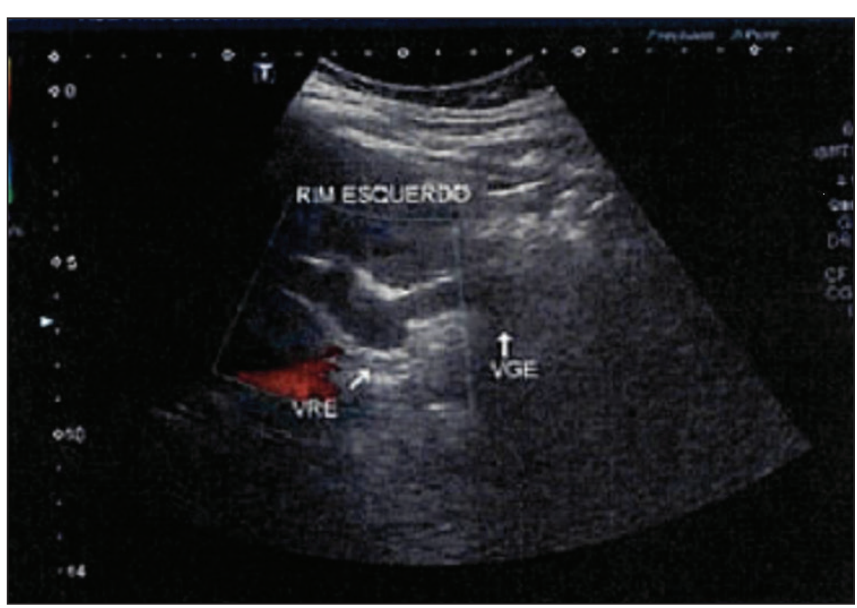

Figura 2. Veias renal e gonadal esquerdas sem fluxo à dopplerfluxometria, confirmando o diagnóstico de trombose de veias renal e gonadal esquerdas

e sem proteinúria significativa. Até o momento, mantém anticoagulação e não apresentou novos episódios de dor em flanco ou de trombose. Tem plano de anticoagulação por tempo prolongado.

\section{Discussão}

Os principais fatores de risco para eventos tromboembólicos na gestação e puerpério são idade maior que 30 ou 35 anos $^{2,5,6,16,18,20,21}$, multiparidade ${ }^{2,5,18,21}$, cesariana (principalmente se de urgência) $)^{2,5-7,16,18,20-22}$, hipertensão específica da gestação ${ }^{5-7,21}$, diabetes gestacional ${ }^{5}$, obesidade $^{2,4,6,7,18-20,22}$, hiperemese gravídica ${ }^{6}$, história pessoal ou familiar de trombose ${ }^{2-5,18}$, hemorragia pós-parto ${ }^{2,5-7,22}$, anemia falciforme ${ }^{2,4,19}$, lúpus eritematoso sistêmico ${ }^{4}$, infecções ou internações na gravidez ${ }^{5,6}$, trabalho de parto prematuro $^{20,22}$, feto morto ${ }^{22}$, gravidez múltipla ${ }^{6,7,20}$, cor negra ${ }^{7,16,19}$, antecedentes de uso de técnicas de reprodução assistida $^{5}$, trombofilia ${ }^{3-5,7,19}$ e tabagismo ${ }^{4-7,19}$. Sendo assim, conhecer os fatores de risco para trombose venosa profunda nas gestantes e puérperas é essencial para avaliar a necessidade de profilaxia e diagnótico ${ }^{5,22}$.

A deficiência de antitrobina, proteína $C$ e proteína $\mathrm{S}$, fator $\mathrm{V}$ de Leiden e mutação do gene da protrombina estão presentes em metade dos casos de tromboembolismo na gravidez e no puerpério. Entretanto, eles estão presentes em $10 \%$ da população ocidental ${ }^{3,4,8}$. Portanto, essa alteração genética aumenta a chance de trombose mas, geralmente, precisa estar associada a outros fatores genéticos ou adquiridos para causar o evento ${ }^{1,4}$. Além disso, nos casos de deficiência de antitrombina, proteína $\mathrm{C}$ e proteína $\mathrm{S}$, o risco de trombose depende também do grau de deficiência ${ }^{1}$.

O risco de desenvolver trombose venosa sempre é maior com esses fatores genéticos quando há história familiar, pois há fatores desconhecidos envolvidos além das mutações ${ }^{1}$. No caso apresentado, apesar de a investigação de trombofilias hereditárias ser negativa, a história familiar é importante, fazendo pensar que existe algum componente genético envolvido. Quanto aos demais fatores de risco, os únicos apresentados pela paciente eram o tabagismo e a multiparidade.

Possivelmente, o fator decisivo para o desenvolvimento da trombose foi a variação anatômica da veia renal esquerda retro-aórtica apresentada pela paciente, que ocorre em 1,7 a 3,4\% dos indivíduos ${ }^{23}$. Sendo assim, a veia pode ter sido comprimida pelo útero gravídico e traumatizada durante o trabalho de parto, gerando as condições necessárias à ocorrência de uma trombose.

Os sinais e sintomas apresentados pela paciente eram compatíveis com os descritos na literatura: dor e enrijecimento do flanco (podendo simular abdome agudo), hematúria microscópica, disfunção renal, proteinúria, febre e alteração da função renal. O diagnóstico não foi aventado de início e só foi pensado depois de excluídos os quadros de pielonefrite e litíase renal.

A TVR esquerda pode levar à trombose da veia gonadal ipsilateral, manifestando-se com edema e dor do grande lábio esquerdo ${ }^{24,25}$. Não há exames de laboratório específicos para a confirmação, e o diagnóstico clínico tem baixa acurácia ${ }^{26}$. No caso de obstrução completa da veia renal, haverá aumento do tamanho do rim com pico em uma semana devido à estase e distensão da cápsula renal ${ }^{11}$. Após, há redução do tamanho e até atrofia. O USG irá evidenciar aumento do tamanho e da ecogenicidade do rim e perda da diferença cortico-medular ${ }^{11}$. A dopplerfluxometria renal pode apresentar ausência de fluxo na veia renal ou fluxo aumentado na seção desobstruída da veia. A sensibilidade do USG é de $85 \%$ e a especificidade é de $56 \%{ }^{11}$.

A TC contrastada é o exame de escolha para o diagnóstico da TVR, tendo sensibilidade e especificidade próximas a $100 \%$. Porém, além da exposição à radiação, 
há exposição a contraste nefrotóxico ${ }^{11}$. A angiografia por ressonância nuclear magnética (RNM) é uma alternativa, mas com sensibilidade e especificidade um pouco menores $^{11}$. O padrão ouro para o diagnóstico é a venografia, pouco utilizada por ser exame invasivo e expor a paciente a radiação e contraste iodado ${ }^{11,13}$.

Pacientes de alto risco, principalmente as que têm mais de um fator de risco, devem receber profilaxia ante e pós-natal ${ }^{1,2,17,21}$. A profilaxia antenatal deve ser realizada de preferência com heparina de baixo peso molecular subcutânea, sendo os benefícios sobre a não facionada: menor risco de trombocitopenia tipo II, pode ser utilizada em dose única diária e menor risco de indução a osteoporose e alergias ${ }^{3,4,21,27,28}$. A dose recomendada para profilaxia é de $40 \mathrm{mg} /$ dia de enoxaparina (heparina de baixo peso molecular) ou 7.500 a 10.000 UI de heparina não-fracionada subcutânea a cada 12 horas (manter o tempo de tromboplastina parcial ativada entre 35 e 40 segundos), de 20 semanas de gestação até 6 semanas após o parto ${ }^{1-5}$. Sugere-se que a heparina de baixo peso molecular seja substituída pela não fracionada na $36^{\mathrm{a}}$ semana ou duas semanas antes da data prevista para o parto, pois a heparina não fracionada tem meia-vida mais curta e pode ser suspensa 12 horas antes do esperado para o parto, cesariana ou analgesia epidural, para evitar sangramentos ${ }^{4,5}$. A heparina deve ser reintroduzida de quatro a seis horas após o parto vaginal ou 6 a 12 horas após cesariana ${ }^{5}$. A heparina não fracionada e a de baixo peso molecular não são excretadas no leite materno e não passam para a placenta. Já a varfarina via oral ultrapassa a barreira placentária e deve ser evitada durante a gravidez, mas é segura durante a lactação ${ }^{3-5}$.

Não é recomendado rastreamento de trombofilias para todas as gestantes, mas sugere-se fazê-lo para as mulheres com história pessoal ou familiar de trombose, parente de primeiro grau com trombofilia hereditária ${ }^{1,3,29}$.

A TVR pode se resolver espontaneamente sem deixar sequelas (disfunção renal e a hipertensão arterial sistêmica) ${ }^{12}$. Para as pacientes com trombose venosa unilateral e assintomática, nenhum tratamento específico está indicado ${ }^{30}$. A decisão do uso de anticoagulantes no seu tratamento deve levar em conta o risco de recorrência e as comorbidades contra o risco de complicações hemorrágicas do tratamento ${ }^{9}$. Atenção principalmente para a heparina de baixo peso molecular nas pacientes com disfunção renal, pois ela pode acumular-se no organismo e aumentar o risco de eventos hemorrágicos ${ }^{11}$.

Pacientes com TVR têm probabilidade aumentada de trombofilia hereditária e devem ser tratadas com anticoagulante oral por três a seis meses e fazer todos os exames de rastreamento para trombofilias hereditárias ${ }^{8,11}$. Essa pesquisa deve ser feita cerca de seis meses após evento de trombose, momento em que deverá ser decidida a continuação ou interrupção da anticoagulação. Antes desse período, os resultados podem não ser muito confiáveis, pois a trombose em si pode alterar alguns fatores ${ }^{8}$.

As principais trombofilias hereditárias em ordem decrescente de prevalência são ${ }^{8}$ : Fator $\mathrm{V}$ de Leiden, Mutação do fator II (protrombina), Deficiência de anitrombina, Deficiência de proteína C, Deficiência de proteína $S$, Disfibrinogenemia e Homocistinúria homozigótica. A anticoagulação deve ser mantida indefinidamente nos pacientes com: evento trombótico ameaçador à vida, trombose de veia cerebral ou visceral, eventos recorrentes, deficiência de antitrobina, homozigose para fator $\mathrm{V}$ de Leiden, trombofilias combinadas ou presença de anticorpos antifosfolípide . $^{8}$

A heparina por tempo prolongado pode levar à osteoporose, devendo ser substituída por varfarina 48 horas após o parto ${ }^{4}$. Para tanto, a heparina deve ser mantida até que o RNI esteja entre 2,0 e 3,02,11,25. No caso de uso de heparina não fracionada subcutânea, sugere-se $15.000 \mathrm{a}$ 30.000 UI 12/12 horas (mantendo-se o tempo de tromboplastina parcial ativada entre 60 e 80 segundos e relação $\mathrm{D} / \mathrm{N}$ entre 1,5 e 2,5 ) ou enoxaparina subcutânea $1 \mathrm{mg} / \mathrm{kg}$ de 12 em 12 horas $^{1-5,12}$. Alguns casos específicos são elegíveis para trombectomia mecânica ou farmacológica (trombólise): falha no tratamento com anticoagulante, complicações como tromboembolismo pulmonar, trombose bilateral, insuficiência renal aguda (trombose bilateral ou rim único), extensão para veia cava inferior, contraindicação ao uso de anticoagulante sistêmico, transplante renal e dor no flanco severa e persistente ${ }^{12}$.

\section{Agradecimentos}

À Vivian Franco (médica hematologista) pelo auxílio na condução do caso e revisão da literatura referente às trombofilias.

\section{Referências}

1. Zotz RB, Gerhardt A, Scharf RE. Inherited thrombophilia and gestational venous thromboembolism. Best Pract Res Clin Haematol. 2003; 16(2):243-59.

2. Greer IA. Epidemiology, risk factors and prophylaxis of venous thromboembolism in obstetrics and gynaecology. Baillieres Clin Obstet Gynaecol. 1997; 11 (3):403-30.
3. Benedetto C, Marozio L, Tavella AM, Salton L, Grivon $\mathrm{S}$, Di Giampaolo F. Coagulation disorders in pregnancy: acquired and inherited thrombophilias. Ann N Y Acad Sci. 2010;1205:106-17.

4. Battinelli EM, Marshall A, Connors JM. The role of thrombophilia in pregnancy. Thrombosis. 2013;2013:516420. 
5. Ralli E, Zezza L, Caserta D. Pregnancy and venous thromboembolism. Curr Opin Obstet Gynecol. 2014;26(6):469-75.

6. Virkus RA, Løkkegaard E, Lidegaard $\varnothing$, Langhoff-Roos J, Nielsen AK, Rothman KJ, et al. Risk factors for venous thromboembolism in 1.3 million pregnancies: a nation wide prospective cohort. PLoS One. 2014;9(5):e96495.

7. Abbasi N, Balayla J, Laporta DP, Kezouh A, Abenhaim HA. Trends, risk factors and mortality among women with venous thromboembolism during labour and delivery: a populationbased study of 8 million births. Arch Gynecol Obstet. $2014 ; 289(2): 275-84$.

8. Seligsohn U, Lubetsky A. Genetic susceptibility to venous thrombosis. N Engl J Med. 2001;344(16):1222-31.

9. Wysokinski WE, Gosk-Bierska I, Greene EL, Grill D, Wiste H, McBane RD 2nd. Clinical characteristics and long-term followup of patients with renal vein thrombosis. Am J Kidney Dis. 2008; 51 (2):224-32.

10. Zucchelli P. Renal vein thrombosis. Nephrol Dial Transplant. 1992;7 Suppl 1:105-8.

11. De Stefano V, Martinelli I. Abdominal thromboses of splanchnic, renal and ovarian veins. Best Pract Res Clin Haematol. 2012;25(3):253-64.

12. Asghar M, Ahmed K, Shah SS, Siddique MK, Dasgupta $P$, Khan MS. Renal vein thrombosis. Eur J Vasc Endovasc Surg. 2007;34(2):217-23.

13. Radhakrishnan J. Renal vein thrombosis and hypercoagulable state in nephrotic syndrome [Internet]. 2013 [cited $2015 \mathrm{Apr}$ 14]. Available from: <http://www.uptodate.com/contents/ renal-vein-thrombosis-and-hypercoagulable-state-in-nephroticsyndrome>

14. Morrissey EC, McDonald BR, Rabetoy GM. Resolution of proteinuria secondary to bilateral renal vein thrombosis after treatment with systemic thrombolytic therapy. Am J Kidney Dis. 1997;29(4):615-9.

15. Llach F. Hypercoagulability, renal vein thrombosis, and other thrombotic complications of nephrotic syndrome. Kidney Int. $1985 ; 28(3): 429-39$.

16. Stein PD, Hull RD, Kayali F, Olson RE, Alshab AK, Meyers FA, et al. Venous thromboembolism in pregnancy: 21 -year trends. Am J Med. 2004;1 17(2):121-5.

17. Heit JA, Kobbervig CE, James AH, Petterson TM, Bailey KR, Melton JJ 3rd. Trends in the incidence of venous thromboembolism during pregnancy or postpartum: a 30-year population-based study. Ann Intern Med. 2005; 143(10):697-706.

18. Macklon NS, Greer IA. The deep venous system in the puerperium: an ultrasound study. Br J Obstet Gynaecol. 1997;104(2):198-200.
19. James AH, Jamison MG, Brancazio LR, Myers ER. Venous thromboembolism during pregnancy and the postpartum period: incidence, risk factors, and mortality. Am J Obstet Gynecol. 2006; 194(5): $1311-5$.

20. Simpson EL, Lawrenson RA, Nightingale AL, Farmer RD. Venous thromboembolism in pregnancy and the puerperium: incidence and additional risk factors from a London perinatal database. BJOG. $2001 ; 108(1): 56-60$.

21. Treffers PE, Huidekoper BL, Weenink GH, Kloosterman GJ. Epidemiological observation of thromboembolic disease during pregnancy and in the puerperium, in 56,022 women. Int J Gynaecol Obstet. 1983;21(4):327-31.

22. Sultan AA, Tata L, West J, Fiaschi L, Fleming KM, Nelson-Piercy $C$, et al. Risk factors for first venous thromboembolism around pregnancy: a population-based cohort study from the United Kingdom. Blood. 2013;121(19):3953-61.

23. Yang C, Trad HS, Mendonça SM, Trad CS. Anomalias congênitas da veia cava inferior: revisão dos achados na tomografia computadorizada multidetectores e ressonância magnética. Radiol Bras. 2013;46(4):227-33.

24. Wang IK, Lee CH, Yang BY, Chang HY, Lin CL, Chuang FR. Lowmolecular-weight heparin successfully treating a nephrotic patient complicated by renal and ovarian vein thrombosis and pulmonary embolism. Int J Clin Pract Suppl. 2005;(147):72-5.

25. Stella N, Rolli A, Catalano A, Udini M. [Simultaneous urokinase perfusion in renal artery and vein in case of renal vein thrombosis]. Minerva Cardioangiol. 2001;49(4):273-8. Italian.

26. Ricci MA, Lloyd DA. Renal venous thrombosis in infants and children. Arch Surg. 1990; 125(9): 11 195-9.

27. Sanson BJ, Lensing AWA, Prins HM, Ginsberg JS, Barkagan ZS, Lavenne-Pardonge $E$, et al. Safety of low-molecular-weight heparin in pregnancy: a systematic review. Thromb Haemost. 1999;81(5):668-72.

28. Douketis JD, Ginsberg JS, Burrows RF, Duku EK, Webber CE, Brill-Edwards $P$. The effects of long-term heparin therapy during pregnancy on bone density. A prospective matched cohort study. Thromb Haemost. 1996;75(2):254-7.

29. Bates SM, Greer IA, Pabinger I, Sofaer S, Hirsh J; American College of Chest Physicians. Venous thromboembolism, thrombophilia, antithrombotic therapy, and pregnancy: American College of Chest Physicians Evidence-Based Clinical Practice Guidelines (8th Edition). Chest. 2008;133(6 Suppl):844S-886S.

30. Wu CH, Ko SF, Lee $\mathrm{CH}$, Cheng BC, Hsu KT, Chen JB, et al. Successful outpatient treatment of renal vein thrombosis by lowmolecular weight heparins in 3 patients with nephrotic syndrome. Clin Nephrol. 2006;65(6):433-40. 